\title{
Cerebellar Infarction in Adolescent Males Associated With Acute Marijuana Use
}

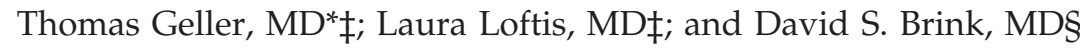

\begin{abstract}
Objective. To demonstrate the clinical characteristics, radiologic findings, and neuropathological features of tetrahydrocannabinol-related posterior fossa ischemic stroke in adolescent patients.

Design. A retrospective case and chart review of 3 cases encountered at a tertiary care institution over a span of 5 years.

Setting. Inpatient and intensive care hospitalization units managing children and adolescents.

Subjects. Male adolescent patients with ischemic cerebellar stroke after use of marijuana.

Diagnostic Investigations. Computed tomography brain scans (3 subjects), magnetic resonance imaging brain study (1 subject), cerebral arteriography (1 subject), cerebellar biopsy (1 subject), and necropsy ( 2 subjects).

Results. Three adolescent males had similar presentations of headache, fluctuating level of consciousness or lethargy, visual disturbance, and variable ataxia after self-administration of marijuana. They developed primary cerebellar infarctions within days after the exposure that could not be attributed to supratentorial herniation syndromes and only minimally involved brainstem structures.

Conclusions. Episodic marijuana use may represent a risk factor for stroke in childhood, particularly in the posterior circulation. Early recognition of the cerebellar stroke syndrome may allow prompt neurosurgical intervention, reducing morbidity. Pediatrics 2004;113:e365-e370. URL: http://www.pediatrics.org/cgi/content/full/113/4/e365; ischemic stroke, cerebral blood flow, marijuana, adolescent drug use.
\end{abstract}

ABBREVIATIONS. THC, tetrahydrocannabinol; CT, computed tomography; CSF, cerebrospinal fluid.

$\mathrm{T}$ The incidence of stroke in childhood and adolescence is very low, at $\sim 6$ cases per 100000 children per year, with ischemic events slightly more common than hemorrhagic causes. Mortality with ischemic stroke is less common in pediatric than in adult patients and is more likely to occur with a massive embolus from the heart to the brain rather than from atherothrombotic occlusion. ${ }^{1}$ The cause of death is typically herniation from brain edema. Among ischemic strokes, $20 \%$ are related to cardiac causes, $70 \%$ are related to cerebral vascular disor-

From the Departments of *Neurology, $\ddagger$ Pediatrics, and §Pathology, St Louis University School of Medicine, St Louis, Missouri.

Received for publication Aug 8, 2003; accepted Nov 26, 2003.

Reprint requests to (T.G.) St Louis University School of Medicine, 1465 S Grand Blvd, St Louis, MO 63104. E-mail: gellertj@slu.edu

PEDIATRICS (ISSN 0031 4005). Copyright (C) 2004 by the American Academy of Pediatrics. ders, and the remainder are related to hematologic disorders, coagulopathies, metabolic disorders affecting cerebral perfusion, neurocutaneous disorders, and occasionally drug- or toxin-induced vasospasm or vascular inflammation. ${ }^{2}$

We encountered 3 adolescent cases of ischemic stroke involving the posterior fossa circulation exclusively after the illicit use of marijuana by adolescent males. One of the cases has been reported in the neurosurgical literature, but we present 2 additional cases with similar presentations, which were ultimately fatal, and include autopsy results of the cerebri. This retrospective case study was approved by the Institutional Review Board of St Louis University as exempt under 45 Code of Federal Regulations 46.101(b) Category 4.

\section{CASE REPORTS}

\section{Case 1}

A 16-year-old black male presented to the emergency department for sudden onset of severe headache, photophobia, rightsided weakness and numbness, and inability to use his lower extremities to walk. He had slurred speech and appeared drunk according to the family's report. His medical history was significant for mild asthma, for which he was on no medications. He reported no antecedent trauma and admitted smoking marijuana the day before admission. Family medical history was noncontributory.

His initial vital signs were normal with the exception of mild systolic hypertension (140/80 mm Hg). His physical examination was unremarkable. His neurologic examination demonstrated that he was oriented to person, place, and time, but he was drowsy. He had slurred speech but otherwise intact cranial nerves II-XII. He was able to move all extremities well and had normal muscle tone. His gait and coordination were not tested because of his drowsiness. His laboratory studies consisting of serum chemistries, liver enzyme function tests, coagulation studies, and complete blood count all were normal. A urine drug screen was positive for tetrahydrocannabinol (THC) metabolites (triage panel for drugs of abuse plus tricyclic antidepressants, Biosite, San Diego, CA). This screen is an immunoassay used for the qualitative determination of the presence of the major metabolites of drugs of abuse (phencyclidine, benzodiazepines, cocaine metabolite, amphetamines, THC, opiates, and barbiturates) and tricyclic antidepressants in urine. His serum was negative for ethanol, salicylates, and acetaminophen.

At reexamination in the emergency department, he was very hard to arouse and given a Glasgow coma score of 6 (E1, V1, M4). A computed tomography (CT) brain scan demonstrated small third and lateral ventricles and no masses, midline shifts, or hemorrhages (Fig 1 A). A lumbar puncture demonstrated the cerebrospinal fluid (CSF) to have normal chemistries and no red or white blood cells. Culture of the CSF was reported later as sterile.

He was admitted for observation as a complicated migraine and placed on intravenous fluids and given ketorolac for pain. Over the course of the first hospital day, the patient's mental status and physical examination continued to fluctuate between alert and communicative on the telephone with friends, to con- 

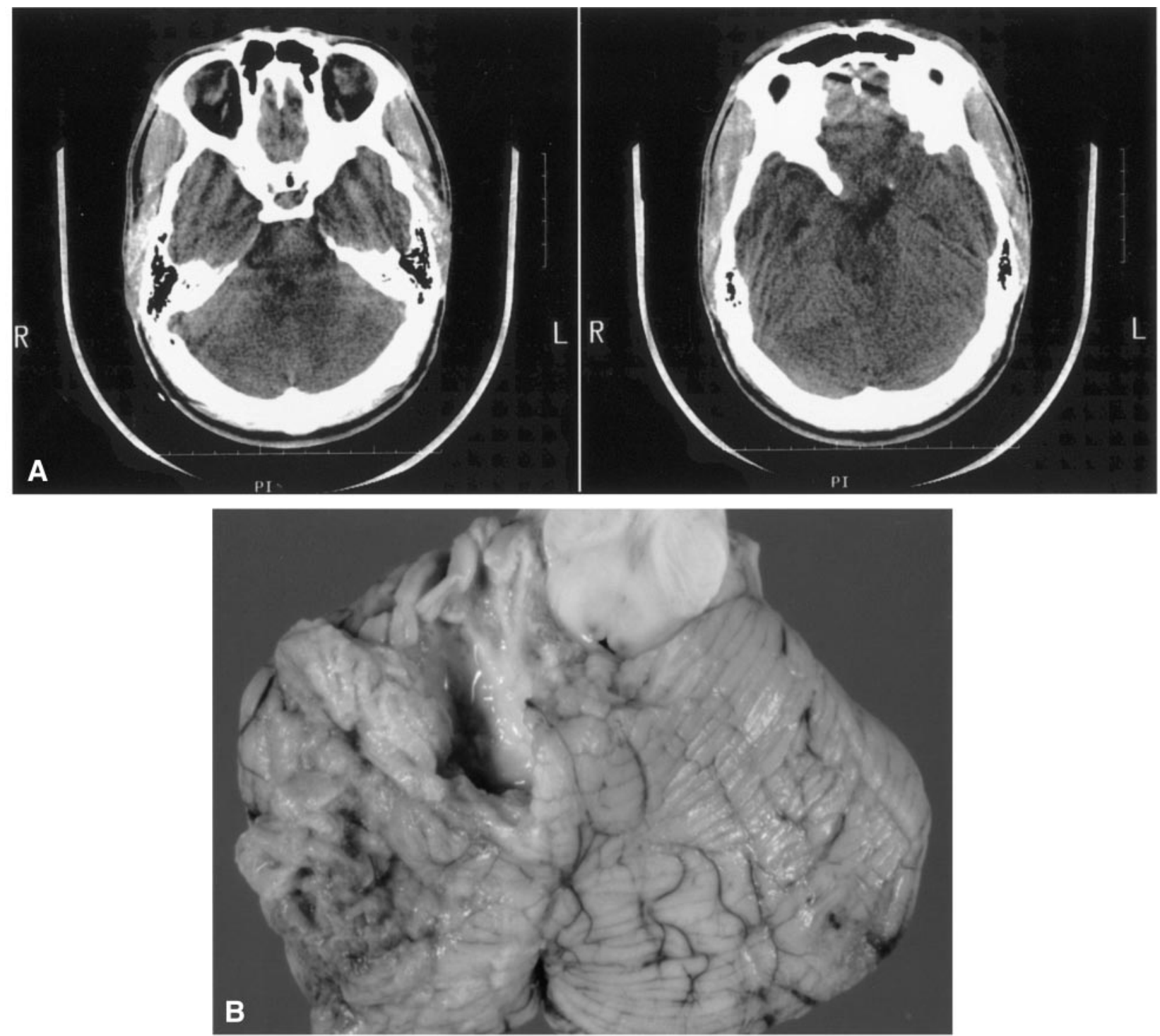

Fig 1. A, CT brain scan of patient 1, initially interpreted as normal but suggestive of an increased attenuation over the right lateral superior cerebellum. B, Superior view of cerebellum and brainstem of patient 1 showing left superior cerebellar acute infarct characterized by marked softening and friability with tissue disruption and minimal associated acute hemorrhage. A smaller infarct is visible as a focus of discoloration in the right cerebellum (4 o'clock, at edge).

fused, dysarthric, and somnolent. A magnetic resonance imaging scan was planned for the following day. Approximately 22 hours after admission, he was found to be completely unresponsive and proceeded to cardiopulmonary arrest. He was shortly thereafter pronounced dead.

\section{Pathology}

At autopsy, the 1220-g brain (age-matched reference range: 1293-1507 g) showed mild diffuse cerebral edema with gyral flattening and sulcal narrowing. The cerebellum showed bilateral acute infarcts, involving the distribution of both superior cerebellar arteries, more prominent on the left (Fig $1 \mathrm{~B}$ ). The left superior cerebellar infarct showed focal early cavitation/liquefaction. Histologically, the cerebellar infarcts demonstrated petechial perivascular hemorrhage, early infiltration by neutrophils, and no infiltration by macrophages (histologic features of an infarct at least 24 hours old and $<3$ days old $)^{3}$; noninfarcted cerebellar parenchyma showed acute ischemic changes predominantly involving Purkinje cells. No anomalies were present in the cerebral vessels, no hematoma or neoplasm was present, and no viral inclusions or granulomata were present. The general autopsy revealed no intracardiac thrombosis as a potential source of emboli, and the remainder of the autopsy was unremarkable.

\section{Case 2}

A 17-year-old white male with a 3-day history of progressively worsening headache associated with nausea and vomiting but without fever was found unresponsive at home. The evening before his initial symptoms, he had been at a party with friends, at which he had smoked marijuana. After returning from the party (4 days before admission), he had not left the home. One day before admission, he had complained of difficulty seeing and walking, and on the morning of admission, he was disoriented. His medical history was significant for mild asthma, but he was on no medications. He had no recent history of trauma and had just passed a military entrance physical examination. By report, he occasionally smoked marijuana and occasionally drank alcohol.

At emergency medical services arrival, he was found to be pulseless and apneic. He was intubated, and cardiopulmonary resuscitation was begun. At arrival to a community hospital, he remained pulseless and apneic, with cardiopulmonary resuscitation continuing for several minutes until return of spontaneous circulation. Initial laboratory values were only remarkable for a severe metabolic and mild respiratory acidosis, an elevated leukocyte count of $23.3 \times 10^{4} / \mathrm{L}$, with $83 \%$ neutrophils. Urine toxicology screen was negative for drugs of abuse (triage panel for drugs of abuse plus tricyclic antidepressants), and serum was 
negative for ethanol, salicylates, and acetaminophen. He was begun on a continuous infusion of dopamine for hypotension, given ceftriaxone, and then transferred to our facility.

At admission to our facility, a CT of his head (Fig 2 A) showed an ill-defined, hypodense, nonenhancing lesion in the left cerebellar vermis with a slight midline shift, cerebellar edema, and early obstructive hydrocephalus. Poor cerebral gray-white differentiation suggested mild cerebral edema. An abdominal CT was normal.

In the intensive care unit, the patient remained hypotensive and unresponsive to pain, with a Glasgow coma score of 3 . His pupils were nonreactive and dilated, and doll's eye responses and corneal reflexes were absent. Physical examination showed a welldeveloped white male with no signs of external trauma. Cardiovascular examination revealed sinus rhythm, no murmurs, and palpable pulses. His abdomen was slightly distended with hypoactive bowel sounds; no hepatosplenomegaly was appreciated. The physical examination was otherwise unremarkable. Additional laboratory studies at the time of admission included normal values for prothrombin time, international normalized ratio, activated partial thromboplastin time, alanine aminotransferase, total bilirubin, and erythrocyte sedimentation rate; an antinuclear antibody assay was negative.

A ventriculostomy was placed and demonstrated intracranial pressures $>60 \mathrm{~cm} \mathrm{H}_{2} \mathrm{O}$ (reference range: 0-20 $\mathrm{cm} \mathrm{H}_{2} \mathrm{O}$ ) despite near-constant draining and modest hyperventilation. Examination of the CSF showed no white or red blood cells and normal glucose and protein values. Within a few hours, the patient developed diabetes insipidus. Despite increasing inotropic support, the pa-
Fig 2. A, CT of the head of patient 2 demonstrating hypodensity in left cerebellar posterior vermis and ventricular enlargement of temporal horns. B, Section of cerebellum and brainstem of patient 2 showing acute infarct in left hemisphere (right side of image). The infarct is softened and friable, with focal acute hemorrhage, and measures $\sim 3.5 \mathrm{~cm}$ in greatest dimension.
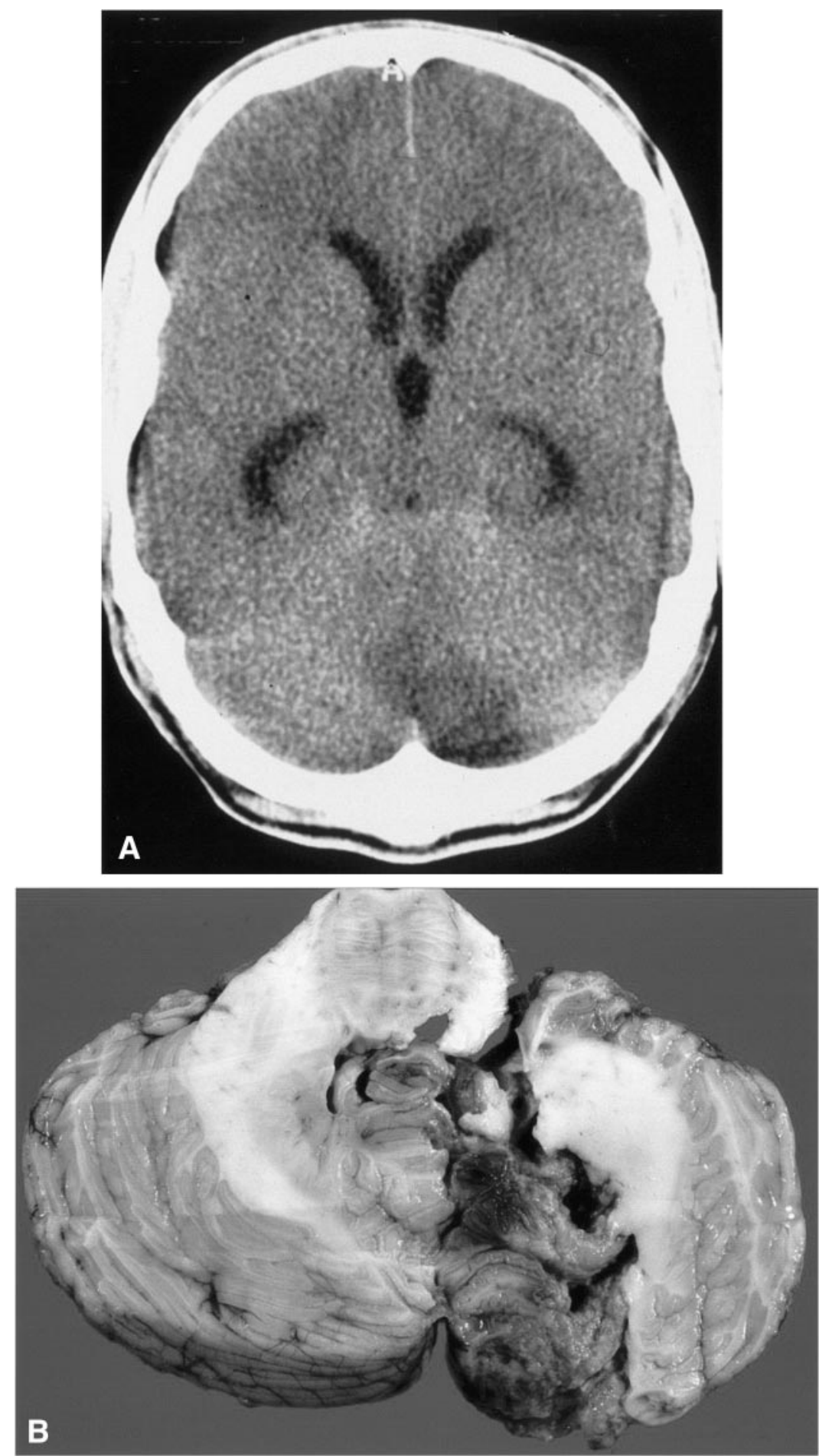
Fig 3. Magnetic resonance image of the head of patient 3 showing $\mathrm{T} 2$ prolongation of signal from both the anterior and posterior right cerebellar cortex as well as localized edema displacing the upper fourth ventricle.

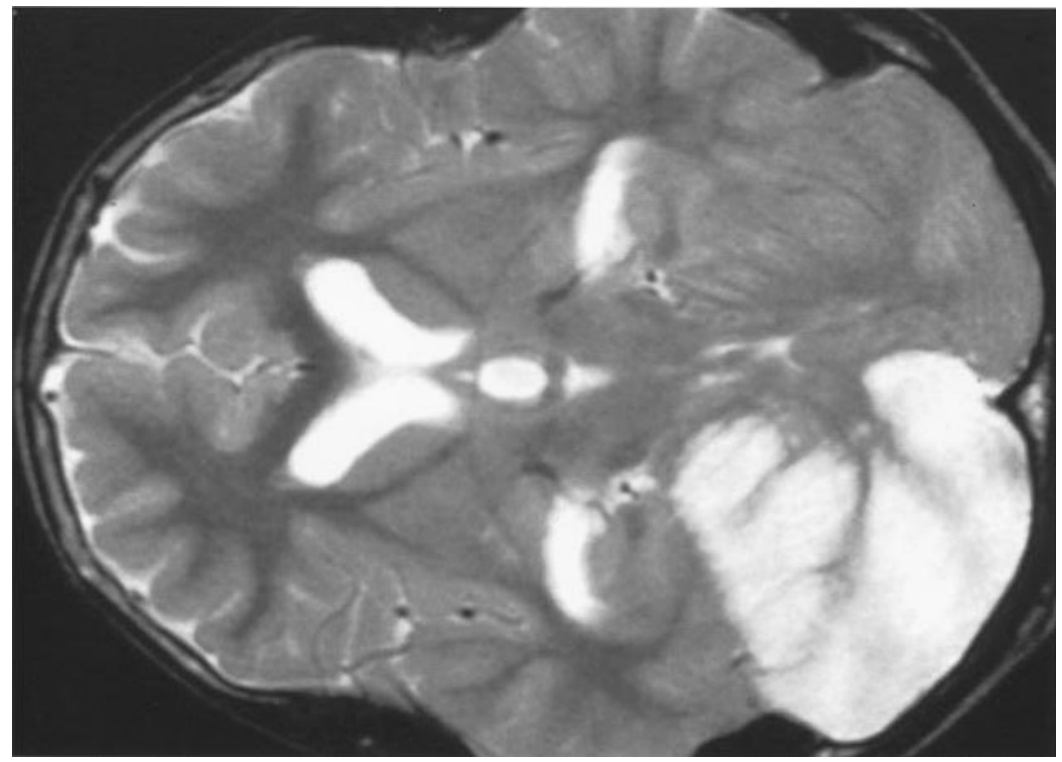

tient's hemodynamic status continued to worsen, and he died 12 hours after admission.

\section{Pathology}

At autopsy, the 1660-g brain (age-matched reference range: 1416-1516 g) showed moderate diffuse cerebral edema with gyral flattening, sulcal narrowing, and compression of the lateral ventricles. The left cerebellum showed asymmetric tonsillar herniation and an acute infarct (Fig 2 B). The infarct contained foci of acute hemorrhage, although no hematoma was present. Histologically, the cerebellar infarct showed focal hemorrhage and infiltration by neutrophils and macrophages (histologic features of an infarct $\sim 3-7$ days old) ${ }^{3}$; noninfarcted cerebellar and cerebral parenchyma showed acute ischemic changes with eosinophilic neurons and cytotoxic edema. No anomalies were present in the cerebral or cerebellar vessels, no neoplasm was present, and no viral inclusions or granulomata were present. The general autopsy revealed no intracardiac thrombosis as a potential source of emboli, and the remainder of the autopsy was unremarkable.

\section{Case 3}

We previously presented a case of biopsy-proven cerebellar infarction involving multiple branch cerebellar arterial territories with a benign cerebral arteriogram in a 15 -year-old male that was temporally related to heavy marijuana use, confirmed by toxicologic study. ${ }^{5}$ This patient presented with a 3-day history of headache, nausea, and unsteadiness of gait after a binge of marijuana smoking. At presentation, he was slightly lethargic and nauseated and had truncal and appendicular ataxia, more prominent on the right side. Although he had some posterior fossa localized mass effect from a right cerebellar multivessel infarct (see Fig 3), he did not develop any cardiopulmonary compromise. He survived and was left with only mild, right-sided dysdiadochokinesia. Extensive evaluations for infectious and noninfectious vasculitides, for coagulopathies, and for cardiac source of emboli were negative.
The patient underwent a diagnostic cerebellar needle biopsy. Histologically, this biopsy revealed infiltration by neutrophils and macrophages (histologic features of an infarct $\sim 3-7$ days old), ${ }^{3}$ which was temporally consistent with his exposure to cannabis.

\section{DISCUSSION}

These 3 adolescent males all developed an acute syndrome of gait instability, headache, cerebellar dysfunction, altered mental status, and variable dysphagia/dysarthria, with evidence of brainstem dysfunction in the 2 subjects who died (Table 1). The clinical and pathologic findings in all 3 cases were related to ischemic infarction in the distribution of multiple distinct cerebellar arterial vessels, which were primary and specifically unrelated to cerebellar herniation from any supratentorial process. The neuropathology did not identify primary tonsillar herniation, which would have been present in a supratentorial process such as diffuse hypoxic-ischemic encephalopathy.

Each of the 3 cannabis-associated cases of cerebellar infarction was confirmed by biopsy ( 1 case) or necropsy ( 2 cases) and showed inflammatory cellular reactions characteristic of a 1- to 3-day-old infarct (case 1) or a 3- to 7-day-old infarct (cases 2 and 3). ${ }^{3}$ In all 3 cases, the central nervous system infarction was acute and localized to cerebellum. Multiple cerebellar arterial distributions were involved in the 3 cases. Brainstem compromise caused by cerebellar and cerebral edema led to death in the 2 fatal cases. For a

TABLE 1. Cerebellar Infarction Patients

\begin{tabular}{|c|c|c|c|}
\hline & 1 & 2 & 3 \\
\hline Age, y & 16 & 17 & 15 \\
\hline Gender & Male & Male & Male \\
\hline Symptoms & $\begin{array}{c}\text { Headache, weakness, } \\
\text { dysarthria, and } \\
\text { visual impairment }\end{array}$ & $\begin{array}{l}\text { Headache, nausea, disorientation, } \\
\text { and visual impairment }\end{array}$ & $\begin{array}{l}\text { Headache, nausea, dizziness, } \\
\text { and ataxia }\end{array}$ \\
\hline Marijuana use & + & + & + \\
\hline $\begin{array}{l}\text { Urine screen for } \mathrm{THC} \\
\text { metabolites }\end{array}$ & + & - & + \\
\hline Neurologic exam & Lethargic, dysarthric & Comatose & Conscious, ataxic \\
\hline Diagnostic method & Autopsy & Autopsy & Biopsy \\
\hline
\end{tabular}


reference of arterial territories of the cerebellum, see ref 6.

None of the patients had pathologic evidence of an embolic source, coagulopathy, or infectious or noninfectious vasculitis, nor was there arteriographic evidence of a systemic predisposition to stroke in the sole surviving patient. However, each subject had a history of acute exposure to marijuana and/or laboratory confirmation of such exposure. The failure to identify marijuana in the second case presented may reflect the 4-day delay between use (based on the history) and testing. The assay used (triage drugs of abuse panel plus tricyclic antidepressants) is an immunoassay for the qualitative determination of the presence of major metabolites of drugs of abuse including 11-nor- $\delta$-9-THC-9-carboxylic acid (11-carboxy-THC), a marijuana metabolite. Although the urinary elimination half-life of 11-nor- $\delta$-9-THC-9carboxylic acid averages 3.0 days, with a range of 0.8 to 9.8 days in heavy smokers of marijuana, ${ }^{7}$ the urine drug screen immunoassay for this metabolite can be negative as early as 3 days after marijuana smoking, particularly in nonheavy and nonchronic users (C. Long, PhD, verbal communication, 2003). Because the patient had recently undergone a military physical examination and laboratory evaluation, and no drugs of abuse were detected at that time, we believe the patient to be an episodic marijuana user. More sensitive assays for marijuana metabolites were not performed in this case, although hair analysis was attempted (unfortunately, the specimen was lost or destroyed in shipping).

The major psychoactive ingredient of marijuana is $\delta$-9-THC. It is lipid soluble with a high volume of distribution $(8.9 \pm 4.2 \mathrm{~L} / \mathrm{kg})$ and a half-life of $32 \pm 12$ hours. ${ }^{8}$ Smoking 1 marijuana cigarette delivers a dose of 2.5 to $5 \mathrm{mg}$ of $\delta$-9-THC. 7 Many other ingredients (psychoactive and nonpsychoactive) are present in cannabis smoke at lesser concentrations and/or lesser potencies than $\delta$-9-THC. Many of these other ingredients have other pharmacologic activities, typically involving the central nervous system and including sedative, analgesic, and anticonvulsant effects. ${ }^{9}$

Marijuana usage has many acute effects including dizziness, impaired balance, postural hypotension, and, at high doses, bradycardia and general hypotension. ${ }^{9}$ Marijuana-associated stroke has been reported in the literature, $, 4,5,10-15$ with some reports being more convincing than others. Patients reported have ranged from 15 to 34 years old and have been exclusively male (including the cases described herein). Several of the reported cases involved infarcts in the distributions of multiple vessels, similar to our cases. Of note, the 3 pediatric cases we have seen were cerebellar rather than cerebral strokes, in contrast to the adult cases reported. Our cases involved large infarcts in regions of $>1$ cerebellar vessel, in contrast to the vast majority of cerebellar strokes, which are typically in the distribution of a single vessel. ${ }^{16,17}$

The neuropharmacologic literature regarding THC generally describes neuroprotective effects from oxidant injury at least in tissue culture ${ }^{18,19}$ as well as therapeutic effects including analgesia, ocular hypotension, and antiemesis. ${ }^{18}$ In a rat model of focal cerebral ischemia, synthetic cannabinoid agonists have been reported to reduce infarct volume. ${ }^{20}$

Nonetheless, the previous reports of stroke and these cases may suggest a more complex effect of cannabinoids. In previously reported stroke cases theorizing pathogenesis, vasospasm and hypotension have been invoked. However, such theories do not explain the apparent proclivity for partial posterior circulation strokes in young patients. Mathew and Wilson ${ }^{21,22}$ have studied regional cerebral blood flow patterns in the setting of numerous drugs of abuse, including marijuana. They used a ${ }^{133} \mathrm{Xe}$ inhalation technique in which clearance of the isotope was measured for 10 minutes by detectors mounted in a helmet worn by the subjects. They found no difference between regional cerebral blood flow of chronic marijuana users and nonusers; unfortunately, their measurements did not include the posterior fossa. Mathew et $\mathrm{al}^{23}$ later used the ${ }^{133} \mathrm{Xe}$ inhalation technique to analyze the acute changes of cerebral blood flow in association with marijuana smoking. In that study, experienced smokers showed an acute increase in cerebral blood flow after marijuana smoking, a change similar to that seen in experienced smokers smoking a placebo cigarette. However, in inexperienced smokers, there was an acute decrease in cerebral blood flow significantly greater than that associated with placebo in inexperienced smokers. Additionally, regardless of the changes in cerebral blood flow observed, the experienced smokers in that study had a significantly lower baseline cerebral blood flow than inexperienced smokers.

We were able to obtain a history of intermittent or "binge" use of marijuana in 2 of our patients (cases 1 and 3 ) and could not get an adequate history of pattern of use in the other case. Nonetheless, we recognize that such a history from the patient and family may be unreliable and cannot be verified. If the subjects were truly episodic users, it is conceivable that cerebellar blood flow could have been compromised. Thus, although the specifics of the effects of marijuana on cerebral perfusion have not been elucidated fully, it is clear that marijuana use does have effects on cerebral blood flow.

\section{CONCLUSIONS}

From the literature, it is clear that marijuana use can cause systemic hypotension, impair peripheral vasomotor reflexes, and may alter central nervous system blood flow and cerebral vascular autoregulation. Marijuana use has been associated with stroke in adults, but acute central nervous system infarction related to marijuana use is not well described in children. Although the mechanism of neurologic injury and its localization to the posterior circulation in these cases remains uncertain, our observation of acute cerebellar infarction in 3 adolescents shortly after marijuana use suggests that this drug may contribute to cerebellar vascular injury, possibly by causing vasospasm, especially in the inexperienced or episodic user, resulting in cerebellar ischemia. The 
cerebellum may be more susceptible to ischemia, because it is a region without rich collateral circulation.

Alternative explanations for ischemic stroke include contamination of marijuana with other substances (unlikely in that the 3 cases we have seen occurred over a 5-year period) and random cerebellar strokes coincident with but unrelated to marijuana use (unlikely given the similarities of our 3 cases in presentation, history, age, gender, and pathologic findings).

Early recognition of cerebellar stroke is essential for prompt neurosurgical intervention to prevent lethal brainstem functional compromise by relieving cerebellar edema. The literature indicates that the mortality rate in cerebellar stroke dramatically increases with the interval from presentation to diagnosis. ${ }^{17}$ If a cerebellar infarction produces a mass effect with signs of brainstem compression or herniation, a surgical decompressive procedure may be necessary in such patients. ${ }^{24,25}$ Although reported strokes associated with marijuana use are few, marijuana use may represent a genuine risk factor for stroke, especially in the adolescent and particularly in the cerebellar circulation.

\section{REFERENCES}

1. De Veber, Roach ES, Riela AR, Wiznitzer M. Stroke in children: recognition, treatment, and future directions. Semin Pediatr Neurol. 2000;7: 309-317

2. Pavlakis SG, Gould RJ, Zito JL. Stroke in children. Adv Pediatr. 1991;38: 151-179

3. Garcia JH, Anderson ML. Circulatory disorders and their effects on the brain. In: Davis RL, Robertson DM, eds. Textbook of Neuropathology. Second ed. Baltimore, MD: Williams \& Wilkins; 1991:621-718

4. Brink D, Manepalli A, Geller T, Vogler C. Cerebellar infarction associated with marijuana use in adolescent children. Mod Pathol. 2001;14:5P

5. White D, Martin D, Geller T, Pittman T. Stroke associated with marijuana abuse. Pediatr Neurosurg. 2000;32:92-94

6. Laurent T, Moulin T, Bogousslavsky J, Duvernoy H. Arterial territories of human brain: brainstem and cerebellum. Neurology. 1996;47: 1125-1135
7. Baselt RC. Disposition of Toxic Drugs and Chemicals in Man. Fourth ed. Foster City, CA: Chemical Toxicology Institute; 2000:812-817

8. Jaffe JH. Drug addiction and drug abuse. In: Gilman AG, Rall TW, Nies AS, Taylor P, eds. Goodman and Gilman's The Pharmacologic Basis of Therapeutics. Eighth ed. New York, NY: Pergamon Press; 1990:522-573

9. Brust JCM. Marijuana. In: Neurological Aspects of Substance Abuse. Woburn, MA: Butterworth-Heinemann; 1993:131-148

10. Mohan H, Sood GC. Conjugate deviation of the eyes after Cannabis indica intoxication. Br J Ophthalmol. 1964;48:160-161

11. Cooles P, Michaud R. Stroke after heavy cannabis smoking. Postgrad Med J. 1987;63:511

12. Zachariah SB. Stroke after heavy marijuana smoking. Stroke. 1991;22: 406-409

13. Barnes D, Palace J, O'Brien MD. Stroke following marijuana smoking. Stroke. 1992;23:1381

14. Lawson TM, Rees A. Stroke and transient ischaemic attacks in association with substance abuse in a young man. Postgrad Med J. 1996;72: 692-693

15. McCarron M, Thomas AM. Cannabis and alcohol in stroke. Postgrad Med J. 1997;73:448

16. Amarenco P, Hauw JJ, Gautier JC. Arterial pathology in cerebellar infarction. Stroke. 1990;21:1299-1305

17. Macdonell RAL, Kalnins RM, Donnan GA. Cerebellar infarction: natural history, prognosis, and pathology. Stroke. 1987;18:849-855

18. Hampson AJ, Grimaldi M, Lolic M, Wink D, Rosenthal R, Axelrod J. Neuroprotective antioxidants from marijuana. Ann $N$ Y Acad Sci. 200;899:274-282

19. van der Stelt M, Veldhuis WB, Bar PR, Veldink GA, Vliegenthart JFG, Nicolay K. Neuroprotection by delta-9-tetrahydrocannabinol, the main active compound in marijuana, against oubain-induced in vivo excitotoxicity. J Neurosci. 2001;21:6475-6479

20. Nagayama T, Sinor AD, Simon RP, Chen J, Graham SH, Jin K, Greenberg DA. Cannabinoids and neuroprotection in global and focal cerebral ischemia and in neuronal cultures. J Neurosci. 1999;19:2987-2995

21. Mathew RJ, Tant S, Burger. Regional cerebral blood flow in marijuana smokers. Br J Addict. 1986;81:567-571

22. Mathew RJ, Wilson WH. Substance abuse and cerebral blood flow. Am J Psychiatry. 1991;148:292-305

23. Mathew RJ, Wilson WH, Tant SR. Acute changes in cerebral blood flow associated with marijuana smoking. Acta Psychiatry Scand. 1989;79: $118-128$

24. Hornig CR, Rust DS, Busse O, Jauss M, Laun A. Space-occupying cerebellar infarction. Clinical course and prognosis. Stroke. 1994;25: 372-374

25. Koh MS, Goh KY, Tung MY, Chan C. Is decompressive craniectomy for acute cerebral infarction of any benefit? Surg Neurol. 2000;53:225-230 